\title{
Contemporary RNA Therapeutics for Glioblastoma
}

\author{
Kaitlyn Melnick ${ }^{1} \cdot$ Farhad Dastmalchi $^{1} \cdot$ Duane Mitchell $^{1} \cdot$ Maryam Rahman $^{1} \cdot$ Elias J. Sayour $^{1}$ (I)
}

Received: 24 December 2020 / Accepted: 21 May 2021 / Published online: 8 June 2021

(c) The Author(s), under exclusive licence to Springer Science+Business Media, LLC, part of Springer Nature 2021

\begin{abstract}
Glioblastoma (GBM) is the most common primary brain tumor in adults and is universally lethal with a median survival of less than two years with standard therapy. RNA-based immunotherapies have significant potential to establish a durable treatment response for malignant brain tumors including GBM. RNA offers clear advantages over antigen-focused approaches but cannot often be directly administered due to biological instability. This review will focus on utilization of RNA dendritic cell vaccines and RNA nanoparticle therapies in the treatment of GBM. RNA-pulsed dendritic cell vaccines have been shown to be safe in a small phase I clinical trial and RNA-loaded nanoparticle vaccines will soon be underway in GBM patients (NCT04573140).
\end{abstract}

Keywords Dendritic cells · Glioblastoma $\cdot$ Immunotherapy $\cdot$ Nanoparticles $\cdot$ RNA $\cdot$ Vaccine

\section{Introduction}

Despite maximal standard therapy of surgery, radiation, and adjuvant chemotherapy with temozolomide, malignant primary brain tumors such as glioblastoma (GBM) carry a poor prognosis (Stupp et al., 2005). In the setting of resounding success for other tumors, investigations into using immunotherapy to target these tumors has been ongoing for decades. Yet, a durable cure for malignant primary brain tumors remains evasive. There are multiple features of primary brain tumors that present challenges for these therapies including the heterogeneity of tumor cell populations, poor penetration of therapies across the blood brain barrier, relative immunosuppressed state of the affected host, and rapid development of tumoral resistance (Chongsathidkiet et al., 2018; Learn et al., 2006).

Ribonucleic acid (RNA) centered immunotherapeutic strategies for targeting refractory tumors have significant advantages over other therapeutic modalities. Messenger RNA (mRNA) offers flexibility of targeting multiple tumor-specific epitopes without risk of genomic integration

Maryam Rahman and Elias J. Sayour: Co-senior; contributed equally.

Kaitlyn Melnick

Kaitlyn.melnick@neurosurgery.ufl.edu

1 Department of Neurosurgery, University of Florida, Gainesville, FL, USA
(DNA vaccines) and without complexity of HLA restricted epitopes which is often limiting in many peptide vaccine trials (Bonehill et al., 2004). A strength of mRNA is that it can be used across all human leukocyte antigen (HLA) genotypes for presentation by major histocompatibility complex (MHC) class I and II proteins (Bonehill et al., 2004). Furthermore, RNA vaccines have garnered recent interest with the FDA approval of mRNA-based vaccines against COVID19 (Anderson et al., 2020; Sahin et al., 2020). There are two major considerations when developing an mRNA vaccine. Firstly, which mRNA should be used and secondly, how should the mRNA be delivered. The basic premise of this technique is to first isolate/identify and manufacture mRNA specific to tumor cells that encodes for tumor-specific antigens. When presented to the host, the mRNA is translated and the resulting protein will be presented to lymphocytes via antigen-presenting cells. This triggers an antigen-specific immunologic response against the mRNA presented endogenously as peptides allowing the host's immune system to begin identifying and attacking a patient-specific cancer. Understanding these strengths and potential pitfalls are imperative for ongoing research development. The primary limitation of mRNA is its bioactive instability and inability to efficiently enter cells. Therefore, the mRNA must be protected, and this can be done through ex vivo loading into cells, or packaged into a virus-like capsid, or a nanoparticle capable of facilitating cell entry. These delivery strategies 
ensure protection of mRNA to allow eventual translation and antigen presentation.

This review provides a brief overview of RNA-based strategies for immunotherapy against GBM including RNApulsed dendritic cells and RNA-loaded lipid nanoparticles. The concepts behind development of these therapeutics will be explained and current preclinical and clinical data will be evaluated.

\section{RNA-Pulsed Dendritic Cells}

\section{Development}

Dendritic cells are professional antigen-presenting cells that are imperative to activating $\mathrm{T}$ cell mediated immune responses. These cells are of hematopoietic origin and present antigens on MHC I and II complexes for activation of CD8+ and CD4+T lymphocytes, respectively. This activation results in significant downstream activation and proliferation of lymphocytes capable of targeting tumor-specific antigens to elicit a robust immune response. Dendritic cells can be harvested from peripheral blood; however, they are in very low concentration and peripheral blood monocytes may not be inherently capable of eliciting a robust anti-tumoral immune response. Therefore, DCs are typically matured, activated and expanded ex vivo prior to host vaccination. Loading of dendritic cells can be achieved with whole tumor lysate, tumor-specific proteins or mRNA encoding tumor-specific proteins. Techniques for introducing mRNA to dendritic cells include viral transduction, electroporation or liposome transfection. For generation of mRNA pulsed dendritic cell vaccines, electroporation is often utilized to facilitate nucleic acid entry ex vivo whereas liposomes are primarily used for lipid nanoparticle mRNA delivery to dendritic cells in vivo.

\section{Clinical Data}

One of the first studies describing the use of dendritic cells pulsed with unfractionated tumor mRNA against glioma was published in 2002 (Insug et al., 2002). This vaccine demonstrated robust CD4 and CD8 intratumoral immune response in a murine model and the effect was enhanced by administration of interleukin (IL)-12. This finding was replicated in humans; however, the authors noted that tumor-specific tolerance was a barrier to effective therapy in some patients (Kobayashi et al., 2003).

It has been known for over a decade that the cellular matrix protein in human cytomegalovirus phosphoprotein 65 (pp65) is an antigen that is highly sensitive and specific for GBM (Mitchell et al., 2008). Although expressed in only low levels, this protein is found in a large proportion of GBM tumors but not in surrounding normal tissue. Host dendritic cells can be isolated from whole blood and transfected with pp65 mRNA ex vivo. Following transfection, the dendritic cells begin to present pp65 restricted epitopes which can be administered as a cell therapy vaccine to all patients (Fig. 1). The immune response generated by these vaccines can be increased by pre-conditioning the vaccine site with tetanus toxoid. This strategy elicits enhanced DC migration to draining lymph nodes and was found to correlate with survival outcomes in a small phase I clinical trial (Mitchell et al., 2015). It is thought that the tetanus toxoid improved efficacy of the vaccine due to a memory recall response to the toxoid resulting in release of chemokines that facilitate trafficking of dendritic cells to lymph nodes (Mitchell et al., 2015). In this small study, the group treated with the tetanus toxoid and pp65 mRNA pulsed dendritic cell vaccines had improved median overall survival ( $>36.6$ months vs 18.5 months). ATTAC-II is an ongoing follow-up phase II clinical trial to further investigate the efficacy of this vaccine strategy in patients with newly diagnosed GBM (clinicaltrials.gov NCT02465268) (Rahman et al., 2019).

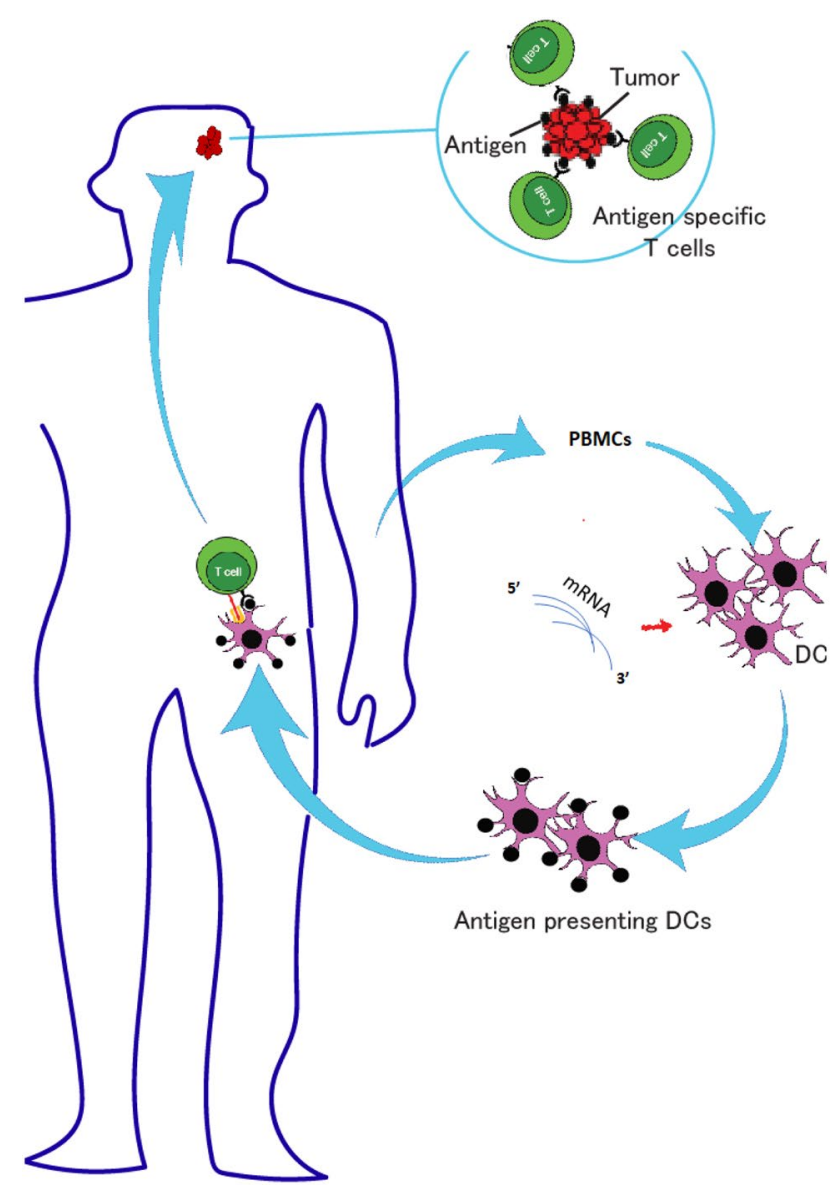

Fig. 1 RNA-pulsed dendritic cell vaccines for GBM 
For patients with recurrent GBM, there is another ongoing trial with RNA-pulsed dendritic cell vaccines (DunnPirio et al., 2017). In this study, RNA is obtained from braintumor stem cells and pulsed into dendritic cells (Dunn-Pirio et al., 2017). This strategy has been investigated in a phase I trial and appears to be safe with a median overall survival of 11 months (Dunn-Pirio et al., 2017). Other antigen targeting strategies for GBM include EGFRvIII, IL13R $\alpha$, Wilms' Tumor 1, HER-2, gp100, and MAGE-1. Some of these are tumor specific (i.e., EGFRvIII) while others can be expressed in normal tissue.

In any immunotherapy strategy, one must consider the potential of excessive autoimmunity induced by the therapy. Although RNA-pulsed DC vaccine therapy appear well tolerated, anaphylactoid reactions have occurred including one patient who had a severe immune reaction including hives, visual changes and headache (Mitchell et al., 2015). However, dendritic vaccines in this context were administered with GM-CSF and further investigation suggested it was this cytokine that precipitated a type I hypersensitivity reaction. Overall, autologous mRNA pulsed DC immunotherapy appears very well tolerated in GBM trials. While mRNA offers flexibility, the process of DC isolation, education and expansion remains tedious. This has led our group to investigate more expedient approaches such as nanoparticle vaccines.

\section{RNA-Loaded Nanoparticles}

\section{Development}

Broadly speaking, nanoparticles are sub-cellular particles that are of organic or inorganic composition. Organic nanoparticles include liposomes, micelles and dendrimers. Inorganic nanoparticles can be made of silica, gold, or iron oxide. Liposomes or lipid nanoparticles have been the most widely employed in delivery of nucleic acids in drug, gene and cancer therapy. A liposome is a lipid bilayer that can be loaded with hydrophilic organic material. The type of RNA loaded into nanoparticles can be mRNA, microRNAs (miRNAs) or small interfering RNAs (siRNAs). mRNA has been extensively studied and in this paradigm, nanoparticles deliver the mRNA to in vivo host dendritic cells which prompts an antigen-specific immune response against tumor expressed epitopes (Fig. 2). A major advantage of the nanoparticle approach is that production of the vaccine is less labor-intensive allowing it to be quicker and cheaper than cell-based approaches. Additionally, RNA is a toll-like receptor agonist which can mediate potent innate response against cancer and synergize with the adaptive response being generated (Sayour et al., 2016, 2018). After local administration (intramuscular) mRNA loaded liposomes mediate a local inflammatory response that brings myeloid cells (i.e., DCs) to site of injection to pick up mRNA loaded particles. Activated DCs can then traffic to regional lymph nodes to educate an adaptive $\mathrm{T}$ cell response. Alternatively, mRNA loaded lipid particles can be injected systemically (i.v.) where they can be naturally filtered by lymphoid organs for myeloid/DC transfection and induction of adaptive $\mathrm{T}$ cell immunity.

\section{Clinical Data}

In a murine model, an intravenously administered liposome made from the cationic lipid 1,2-dioleoyl-3-trimethylammonium-propane (DOTAP) was loaded with whole tumor RNA that effectively activated dendritic cells and resulted in expansion of activated $\mathrm{T}$ cells with improved survival (Sayour et al., 2016, 2018). This effect could be enhanced by addition of immune checkpoint inhibitors such as PD-L1 monoclonal antibodies (Sayour et al., 2016, 2018). Additionally, utilizing RNA nanoparticles as systemic therapy was safe in a spontaneous canine glioma model (Sayour et al., 2018). Modifying liposomes by loading them with iron oxide allows tracking of dendritic cell transfection with

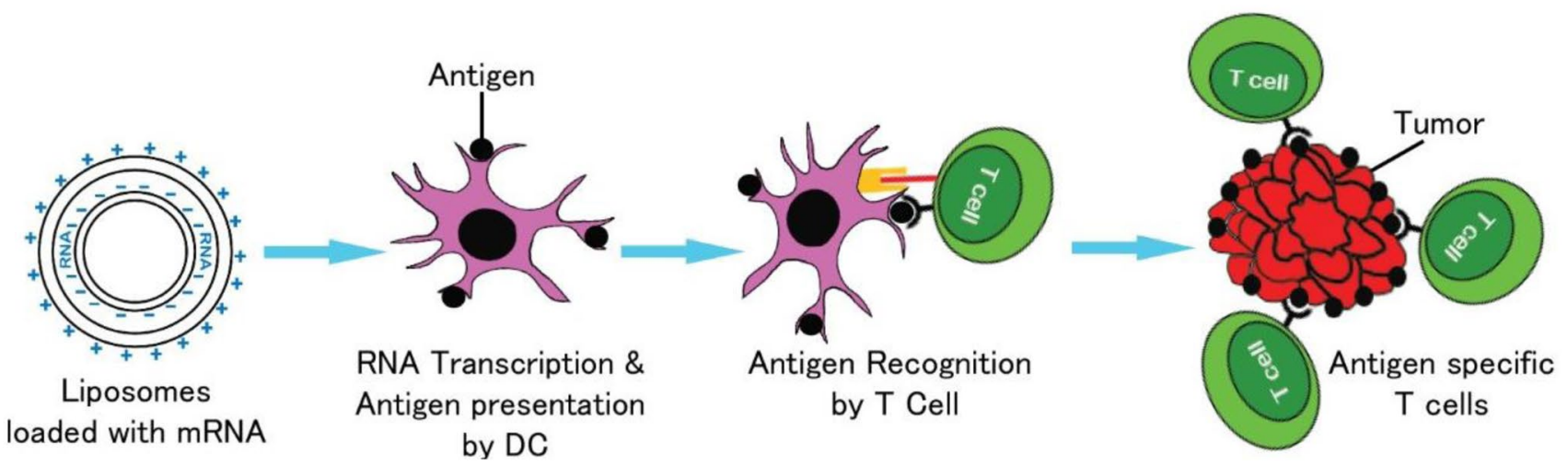

Fig. 2 Illustration of RNA nanoparticle immunotherapy 
MRI. Specifically, in a murine model, MRI signal intensity in host lymph nodes was shown to be an early predictor of anti-tumoral immune response and improved survival (Grippin et al., 2019). This approach may allow early for early recognition of non-responders so that therapy could be rapidly modified. Moreover, nanoparticles may also be loaded with mRNAs encoding the transcription factors such as interferon regulatory factor 5 (IRF5) and its activating kinase IKK $\beta$ (Zhang et al., 2019). These transcription factors result in a shift of tumor associated macrophage cell functions (e.g., M2 to M1 conversion) leading to increased tumor cell immunity and tumor size reduction (Zhang et al., 2019).

A different technique for RNA nanoparticle therapy is to administer microRNAs (miRNAs) or small interfering RNAs (siRNAs). In one design, lipid nanoparticles were utilized to deliver miR-124 which is a microRNA that regulates the signal transducer and activator of transcription 3 (STAT3) pathway thus decreasing inherent tumor immunosuppression (Yaghi et al., 2017). The miR-124 loaded nanoparticle resulted in improved survival in a murine model (Yaghi et al., 2017). Similarly, a solid lipid nanoparticle constructed from components of low-density lipoprotein was linked to PEGylated c-Met siRNA and lead to decreased c-Met expression in tumors with reduced growth (Jin et al., 2011).

A nanoparticle-based approach provides flexibility in nucleic acid delivery and this capacity can be leveraged to administer combination therapies. We have shown that tumor mRNA can be delivered with GM-CSF encoding mRNA for enhanced anti-tumor immunity in preclinical models (Sayour et al., 2016). These foundational studies can be used to develop next-generation approaches for theranostic applications. These applications may be developed with ironoxide reporters for longitudinal reporting and correlation of outcomes with directed particle localization (Grippin et al., 2019). Another potential application under the umbrella of nanotechnology, includes use of hydrogels for immunotherapeutic delivery. A hydrogel is a crosslinked hydrophilic polymer that can suspend organic material including proteins or nucleic acids (Ding et al., 2020). While RNA-loaded hydrogels are still in development for GBM, this therapy has been efficacious in vitro against triple negative breast cancer (Ding et al., 2020). These hydrogels may be leveraged as a scaffold to embed and deliver therapeutics to outcompete the rapid evolution and heterogeneity of GBM.

\section{Conclusion}

In summary, RNA-based immunotherapies for GBM offer numerous advantages over protein-centric therapies. However, due to the biological instability of RNA, these therapies require protection in either a cell or nanocarrier to trigger a robust immune response. Messenger RNA has been used to develop a promising dendritic cell vaccine in a phase I GBM clinical trial with ongoing phase II studies. RNA nanoparticles can be utilized to activate DCs in vivo with robust immune responses in murine and large animal canine models, and human trials will soon be underway (NCT04573140). The flexibility of nanomaterials may offer a unique opportunity to deliver combinatorial therapies that address GBM evolution and heterogenicity in a more facile and commercial formulation.

Author Contributions $\mathrm{KM}$ wrote the manuscript; FD created the art in the figures; DAM reviewed and co-directed the work, MR organized and edited the manuscript; EJS co-wrote and edited the manuscript.

Funding D.A.M. is supported by the National Institutes of Health (NCI R01CA195563, R01CA175517). M.R. is supported by the National Institutes of Health (NINDS K08NS099484). E.J.S. is supported by the Rally Foundation, Hyundai Hope on Wheels, CureSearch for Children's Cancer, Florida Department of Health (Bankhead-Coley and Live Like Bella Cancer Research Programs), and National Institutes of Health (NCI K08CA199224, R37CA251978).

Data Availability Not applicable.

Code Availability Not applicable.

\section{Declarations}

Conflict of interest This manuscript discusses patented technologies by EJS and DAM that are under option to license by iOncologi, Inc., and patents by DAM that have been licensed to Immunomic Therapeutics, Inc., Annias Immunotherapeutics, Inc., and Celldex, Inc.

Ethical Approval Not applicable.

Consent to Participate Not applicable.

Consent for Publication Not applicable.

Research Involving Human Participants and/or Animals Not applicable.

Informed Consent Not applicable.

\section{References}

Anderson, E. J., Rouphael, N. G., Widge, A. T., Jackson, L. A., Roberts, P. C., Makhene, M., et al. (2020). Safety and immunogenicity of SARS-CoV-2 mRNA-1273 vaccine in older adults. New England Journal of Medicine, 383(25), 2427-2438. https://doi. org/10.1056/NEJMoa2028436

Bonehill, A., Heirman, C., Tuyaerts, S., Michiels, A., Breckpot, K., Brasseur, F., et al. (2004). Messenger RNA-electroporated dendritic cells presenting MAGE-A3 simultaneously in HLA class I and class II molecules. The Journal of Immunology, 172(11), 6649-6657.

Chongsathidkiet, P., Jackson, C., Koyama, S., Loebel, F., Cui, X., Farber, S. H., et al. (2018). Sequestration of T cells in bone marrow in the setting of glioblastoma and other intracranial tumors. 
Nature Medicine, 24(9), 1459-1468. https://doi.org/10.1038/ s41591-018-0135-2

Ding, L., Li, J., Wu, C., Yan, F., Li, X., \& Zhang, S. (2020). A selfassembled RNA-triple helix hydrogel drug delivery system targeting triple-negative breast cancer. Journal of Materials Chemistry $B, 8(16), 3527-3533$.

Dunn-Pirio, A., Peters, K., DesJardins, A., Randazzo, D., Friedman, H., Healy, P., et al. (2017). Tumor stem cell RNA-loaded dendritic cell vaccine for recurrent glioblastoma: A phase 1 trial (S41. 004). AAN Enterprises.

Grippin, A. J., Wummer, B., Wildes, T., Dyson, K., Trivedi, V., Yang, C., et al. (2019). Dendritic cell-activating magnetic nanoparticles enable early prediction of antitumor response with magnetic resonance imaging. ACS Nano, 13(12), 13884-13898.

Insug, O., Ku, G., Ertl, H., \& Blaszczyk-Thurin, M. (2002). A dendritic cell vaccine induces protective immunity to intracranial growth of glioma. Anticancer Research, 22(2A), 613-621.

Jin, J., Bae, K. H., Yang, H., Lee, S. J., Kim, H., Kim, Y., et al. (2011). In vivo specific delivery of c-Met siRNA to glioblastoma using cationic solid lipid nanoparticles. Bioconjugate Chemistry, 22(12), 2568-2572.

Kobayashi, T., Yamanaka, R., Homma, J., Tsuchiya, N., Yajima, N., Yoshida, S., et al. (2003). Tumor mRNA-loaded dendritic cells elicit tumor-specific CD8+ cytotoxic T cells in patients with malignant glioma. Cancer Immunology Immunotherapy, 52(10), 632-637.

Learn, C. A., Fecci, P. E., Schmittling, R. J., Xie, W., Karikari, I., Mitchell, D. A., et al. (2006). Profiling of CD4+, CD8+, and $\mathrm{CD} 4+\mathrm{CD} 25+\mathrm{CD} 45 \mathrm{RO}+\mathrm{FoxP} 3+\mathrm{T}$ cells in patients with malignant glioma reveals differential expression of the immunologic transcriptome compared with $\mathrm{T}$ cells from healthy volunteers. Clinical Cancer Research, 12(24), 7306-7315. https://doi.org/ 10.1158/1078-0432.CCR-06-1727

Mitchell, D. A., Batich, K. A., Gunn, M. D., Huang, M.-N., SanchezPerez, L., Nair, S. K., et al. (2015a). Tetanus toxoid and CCL3 improve dendritic cell vaccines in mice and glioblastoma patients. Nature, 519(7543), 366-369.

Mitchell, D. A., Sayour, E. J., Reap, E., Schmittling, R., DeLeon, G., Norberg, P., et al. (2015b). Severe adverse immunologic reaction in a patient with glioblastoma receiving autologous dendritic cell vaccines combined with GM-CSF and dose-intensified temozolomide. Cancer Immunology Research, 3(4), 320-325.
Mitchell, D. A., Xie, W., Schmittling, R., Learn, C., Friedman, A., McLendon, R. E., et al. (2008). Sensitive detection of human cytomegalovirus in tumors and peripheral blood of patients diagnosed with glioblastoma. Neuro-Oncology, 10(1), 10-18.

Rahman, M., Ghiaseddin, A., Yegorov, O., Yang, C., Dechkovskaia, A., Tran, D., et al. (2019). ATIM-15. Sustained complete radiographic response and prolonged systemic immune activation in a patient with MGMT unmethylated midline glioblastoma receiving CMV pp65-LAMP RNA-pulsed dendritic cell vaccines. Neurooncology, 21, vi4.

Sahin, U., Muik, A., Derhovanessian, E., Vogler, I., Kranz, L. M., Vormehr, M., et al. (2020). COVID-19 vaccine BNT162b1 elicits human antibody and TH1 T cell responses. Nature, 586(7830), 594-599. https://doi.org/10.1038/s41586-020-2814-7

Sayour, E. J., De Leon, G., Pham, C., Grippin, A., Kemeny, H., Chua, J., et al. (2016). Systemic activation of antigen-presenting cells via RNA-loaded nanoparticles. Oncoimmunology. https://doi.org/ 10.1080/2162402X.2016.1256527

Sayour, E. J., Grippin, A., De Leon, G., Stover, B., Rahman, M., Karachi, A., et al. (2018). Personalized tumor RNA loaded lipid-nanoparticles prime the systemic and intratumoral milieu for response to cancer immunotherapy. Nano Letters. https://doi.org/10.1021/ acs.nanolett. 8 b02179

Stupp, R., Mason, W. P., van den Bent, M. J., Weller, M., Fisher, B., Taphoorn, M. J., et al. (2005). Radiotherapy plus concomitant and adjuvant temozolomide for glioblastoma. New England Journal of Medicine, 352(10), 987-996. https://doi.org/10.1056/NEJMo a043330

Yaghi, N. K., Wei, J., Hashimoto, Y., Kong, L.-Y., Gabrusiewicz, K., Nduom, E. K., et al. (2017). Immune modulatory nanoparticle therapeutics for intracerebral glioma. Neuro-Oncology, 19(3), 372-382.

Zhang, F., Parayath, N., Ene, C., Stephan, S., Koehne, A., Coon, M., et al. (2019). Genetic programming of macrophages to perform anti-tumor functions using targeted mRNA nanocarriers. Nature Communications, 10(1), 1-16.

Publisher's Note Springer Nature remains neutral with regard to jurisdictional claims in published maps and institutional affiliations. 